\title{
Single Atom Identification of Barium by HAADF-STEM for the New Enriched Xenon Observatory (nEXO)
}

\author{
JB Warren ${ }^{1 *}, \mathrm{M} \mathrm{Chiu}^{2}$ and $\mathrm{D} \mathrm{Su}{ }^{3}$ \\ 1. J. B. Warren, Instrumentation Division, Brookhaven National Laboratory, Upton, NY, USA. \\ 2. M. Chiu, Physics Department, Brookhaven National Laboratory, Upton, NY, USA. \\ 3. D. Su, Center for Functional Nanomaterials, Brookhaven National Laboratory, Upton, NY, USA. \\ * Corresponding author: warren@bnl.gov
}

Successful observation of neutrinoless double beta decay in the nEXO experiment is dependent on ultraefficient detectors because the half-life of ${ }^{136} \mathrm{Xe}$ is projected to be as high as $10^{28}$ years. In $\mathrm{nEXO}$, the detection of a ${ }^{136} \mathrm{Ba}$ daughter from the ${ }^{136} \mathrm{Xe}$ decay provides a way to eliminate extraneous background events. Such "barium-tagging" requires that the $\mathrm{Ba}^{++}$ions generated by ${ }^{136} \mathrm{Xe}$ decay be collected with high efficiency, and then identified with laser induced optical spectroscopy, or resonant ion spectroscopy. Positive identification of $\mathrm{Ba}^{++}$ions by these methods would enable a determination of absolute neutrino mass, a major goal of high energy physics. Since ppm-levels of barium are found in most structural alloys, the sensitivity of these techniques is compromised by barium impurities that mask detection of nEXO-produced barium atoms adhering to the experimental substrate surface.

To eliminate contamination from substrate supports required by spectroscopy, Chiu proposed that a single atomic layer of graphene would be an ideal substrate for barium-tagging since resistive heating can be used to drive off contaminant molecules [1]. Warren further proposed that the graphene films be suspended over an aperture in a $3 \mathrm{~mm}$ diameter disc fitting in a standard electron microscope stage for high resolution single atom identification of barium [2]. Single atom identification has already been achieved at the Brookhaven Center for Functional Nanomaterials using the Hitachi 2700 STEM [3]. With the same instrument, high contrast microscopy methods such as HAADF-STEM could be used to image isolated barium atoms with complimentary EELS and EDX spectra providing positive identification.

To check the feasibility of single Ba atom identification by electron microscopy, single layer graphene films suspended on patterned silicon nitride overlaying standard $3 \mathrm{~mm}$ diameter TEM grids (Ted Pella Inc. Prod. No. 21712-10) and patterned amorphous carbon films (EM Sciences C-flat CF-2/0.5-2C) were coated with a thin layer of metallic barium by vapor deposition from a tantalum boat in an evaporator evacuated to $10^{-7}$ torr. The purity of the film is determined solely by the vapor pressure of barium, which is much greater at $400-500^{\circ} \mathrm{C}$ than any other elemental impurity present in the solid Ba charge used for deposition. Each sample grid was masked so only half was coated with a layer of 2-3 nm of barium. Low magnification SEM imaging showed the boundary between the coated and uncoated sample regions to be a few microns wide. This narrow boundary region was then examined with the Hitachi 2700 STEM at BNL's Center for Functional Nanomaterials to search for single Ba adatoms adhering to the carbon film.

As shown in Fig. 1, individual $\mathrm{BaO}$ adatoms were detected in the boundary between the coated and uncoated areas on both graphene and amorphous carbon films using the HAADF detector. EELS spectra acquired from an array of sub-scans that bracketed target Ba adatoms showed both barium and oxygen peaks. This results from barium's reactivity causing it to bond with oxygen to form $\mathrm{BaO}$ as 
soon as the sample is exposed to atmosphere when it is removed from the evaporator. In the thick deposit regions, STEM images revealed a loose, porous network of barium oxide nanoparticles but at the film boundary the individual adatoms of $\mathrm{BaO}$ were relatively stable and adhered to both graphene and amorphous carbon. At $200 \mathrm{kV}, \mathrm{BaO}$ adatom movement caused by interaction with the STEM probe was evident rendering the samples stable enough only for 10-15 second EELS spectra acquisition. Longer term exposures that would be required for EDX are possible but only if the atom movement can be minimized. Future experiments will be performed at lower $\mathrm{kV}$ to minimize this effect and also to enhance HAADF contrast from high- $Z$ candidate atoms [4].

\section{References:}

[1] M Chiu, Division Seminar Series, Brookhaven National Laboratory (2015).

[2] J Warren and M Chiu, BNL LDRD Proposal (2017).

[3] Y Zhu et al., Nature Matls Lett 8 (2009), p. 808.

[4] This research used resources of the Center for Functional Nanomaterials, which is a U.S. DOE Office of Science Facility, at Brookhaven National Laboratory under Contract No. DE-SC0012704.
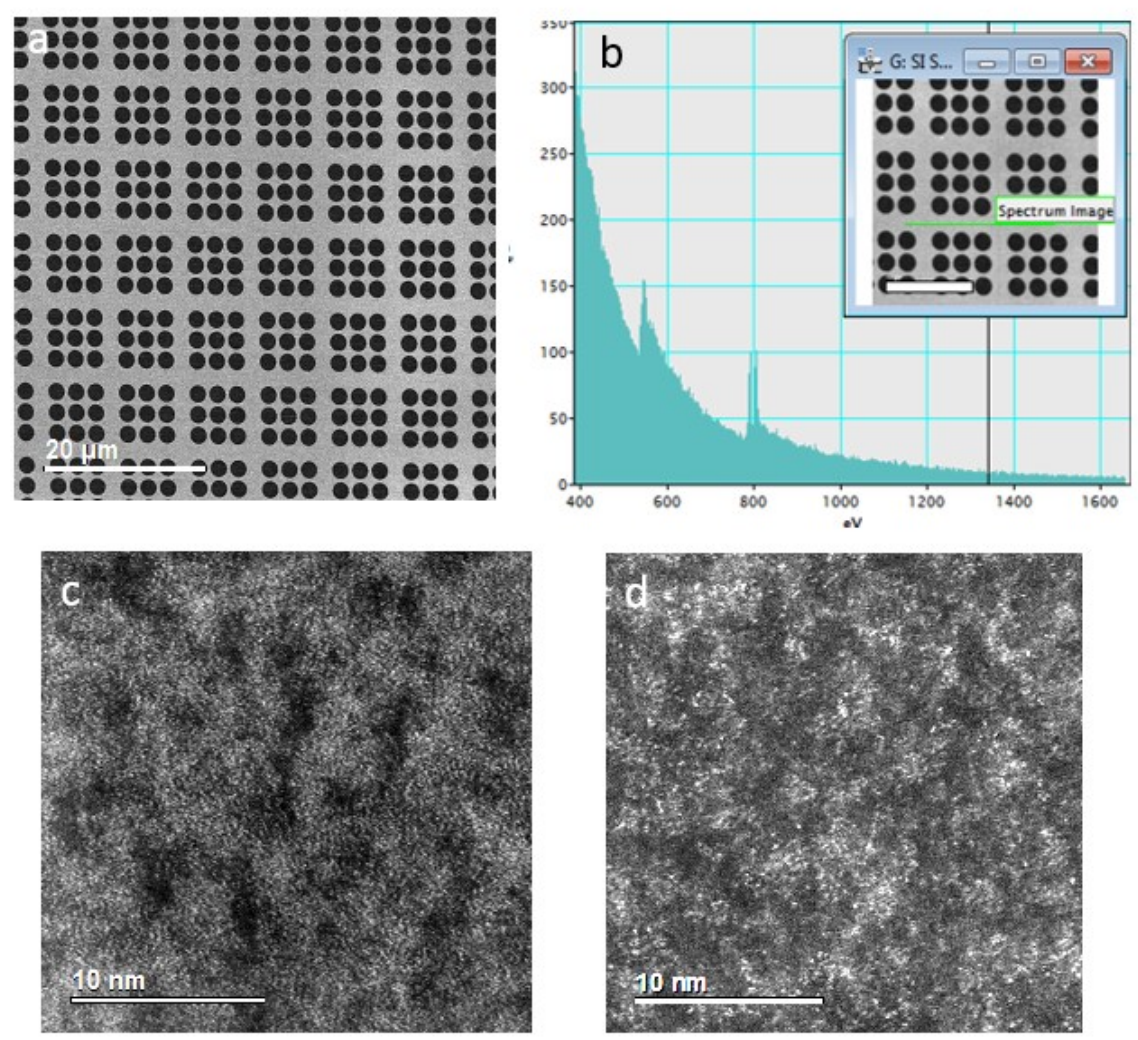

Figure 1. Barium deposition on amorphous carbon C-flat membrane: Fig. 1a shows low magnification SEM image of C-flat, Fig. 1b shows line scan and accompanying EELS spectra with oxygen and barium edges. Fig. 1c shows HAADF STEM image of $\mathrm{BaO}$ film while Fig. 1d shows individual BaO adatoms. 